\title{
Regulation morphological and architectural acclimation of soybean seedlings to shade
}

\author{
Yuanfang Fan ${ }^{1}$, Shenglan $\mathrm{Li}^{1}$, Muhammad Ali Raza ${ }^{1}$, Zhonglin Wang ${ }^{1}$, Beibei Wang ${ }^{1}$, \\ Jiawei Zhang ${ }^{1}$, Xianming Tan ${ }^{1}$, Tingting Tan ${ }^{1}$, Ling-Yang Feng ${ }^{2}$, Xiaoling $\mathrm{Wu}^{1}$, Wen-yu \\ Yang $^{2}$, and Feng Yang ${ }^{2}$ \\ ${ }^{1}$ Sichuan Agricultural University - Chengdu Campus \\ ${ }^{2}$ Sichuan Agricultural University
}

October 21, 2020

\begin{abstract}
Maize shading affects the growth and development of soybean in maize soybean intercropping systems. Two different soybean varieties were (ND12, high shade-tolerant, and GX3, low shade-tolerant) relay intercrop with maize in different (T1, double row, and $\mathrm{T} 2$, single row) relay strip intercropping systems, and the results of the both planting arrangements of strip intercropping system were compared with the sole planting system of soybean. The results showed that shading reduced the content of photosynthetic pigment per unit area of soybean leaf, Fv/Fm, ФPSII, and Fv/Fo of ND12 and GX3 under T1 and T2. Moreover, $\mathrm{T} 1$ and $\mathrm{T} 2$ also decreased the density and size of stomata, leaf thickness, palisade tissue thickness of the tissue, and the size of the chloroplast and starch grains, while increased the thickness of the granum lamellae in both varieties of soybean. Nevertheless, the leaf area, aboveground biomass, photosynthetic capacity, photosynthetic organs measured morphological and architectural indices were found maximum in ND12 compared to GX3 in all planting patterns.
\end{abstract}

Title Page

\section{Soybean seedlings to shade}

\section{Highlight}

1. Shade affects soybean plant growth.

2. Shading increased the thickness of the granum lamellae in both varieties of soybean.

3. The photosynthetic organs measured morphological and architectural indices were found maximum in ND12 compared to GX3 in all planting patterns.

\section{Regulation morphological and architectural acclimation of soybean seedlings to shade}

Yuanfang Fan ${ }^{1,2 \#}$, Shenglan $\mathrm{Li}^{1,2 \#}$, Muhammad Ali Raza ${ }^{1,2}$, Zhonglin Wang ${ }^{1,2}$, Beibei Wang ${ }^{1,2}$, Jiawei Zhang ${ }^{1,2}$, Xianming Tan, Tingting Tan, Lingyang Feng ${ }^{1,2}$, Xiaoling $\mathrm{Wu}^{1,2}$, Wenyu Yang ${ }^{1,2,3^{*}}$, Feng Yang ${ }^{1,2,3^{*}}$

\# These authors contributed equally to this work 
${ }^{1}$ College of Agronomy, Sichuan Agricultural University, Chengdu 611130, People's Republic of China

2 Sichuan Engineering Research Center for Crop Strip Intercropping System, Chengdu 611130, People's Republic of China

${ }^{3}$ Key Laboratory of Crop Ecophysiology and Farming System in Southwest, Ministry of Agriculture, Chengdu 611130, People's Republic of China

${ }^{*}$ Corresponding author.

Post address: College of Agronomy, Sichuan Agricultural University, Huimin Road 211, Wenjiang District, Chengdu 611130, P.R.China

Tel: +86-28-86290960; Fax: +86-28-86290870

Email address:f.yang@sicau.edu.cn;mssiyangwy@sicau.edu.cn

\begin{abstract}
Maize shading affects the growth and development of soybean in maize soybean intercropping systems. Here we reveal that the intercropping systems increased the biomass investment in the stem, whereas it decreased the biomass allocation to leaves and reduced the size of palisade cells and chloroplasts, which ultimately decreased photosynthetic efficiency and biomass accumulation in soybean during the co-growth period of intercrop species in T1. In this study, two different soybean varieties was (ND12, high shade-tolerant, and GX3, low shade-tolerant) relay intercrop with maize in different ( $\mathrm{T} 1$, double row, and $\mathrm{T} 2$, single row) relay strip intercropping systems, and the results of the both planting arrangements of strip intercropping system were compared with the sole planting system of soybean (CK). The results showed that shading increased the plant height and stem biomass ratio of soybeans and reduced the leaf area, specific leaf weight, leaf biomass ratio, and aboveground biomass of soybeans per plant in both soybean varieties under T1 and T2. Meanwhile, compared with CK, shading also reduced the content of photosynthetic pigment per unit area of soybean leaf, the maximum light energy conversion efficiency of photosystem II $\left(F_{v} / F_{m}\right)$, the actual light energy conversion efficiency ( $\Phi$ PSII), and photosynthesis active $\left(F_{v} / F_{o}\right)$ of ND12 and GX3 under T1 and T2. Moreover, T1 and T2 also decreased the density and size of stomata, leaf thickness, palisade tissue thickness, sponge thickness of the tissue, and the size of the chloroplast and starch grains, while increased the thickness of the granum lamellae in both varieties of soybean. Nevertheless, the leaf area, aboveground biomass, photosynthetic capacity, photosynthetic organs measured morphological and architectural indices were found maximum in ND12 compared to GX3 in all planting patterns (T1, T2, and CK). Overall, these findings indicate that soybean plants under intercropping systems make adjustments in their morphology and leaf architect to cope with the shading conditions. However, further studies are required to fully understand the internal signaling and the molecular mechanism regulating soybean morphology and leaf architect in intercropping systems.
\end{abstract}

\title{
KEYWORDS
}

Shade; Photosynthesis; Chlorophyll fluorescence; Leaf structure; Chloroplast

\section{INTRODUCTION}

Soybean is widely cultivated worldwide, and it is one of the most important food crops in developed (USA) and developing (China) countries, especially for oil and protein. It contains numerous functional components, such as amino acids, unsaturated fatty acids, and flavonoids, which play an essential role in human health improvement. However, the current supply of soybean is insufficient to meet the increasing demand of a growing population. With environmental pollution, climate change, population expansion, and urbanization, the global food crisis has intensified. (Foley et al., 2011). China is the world's most populous country with limited arable land. Food security is a major public issue in China. High-input fertilizers and water irrigation 
have aggravated water and air pollution, soil acidification, and erosion in recent years. These serious problems have brought new challenges to food security and sustainability (Du et al., 2018). Therefore, improvement in the production of soybean is necessary.

Intercropping of cereal with legume significantly improve the resource efficiencies and maintain sustainability (Drinkwater, Wagoner, \& Sarrantonio, 1998). For instance, Liu et al. (2018) estimated that maize and soybean in maize soybean strip intercropping system intercepted $5 \%$ and $19 \%$, respectively, more light than sole maize and soybean, explaining the reason for the yield gain of this system over sole cropping systems (Liu et al., 2018). Moreover, researchers have confirmed that maize and soybean are the best partners under intercropping systems, using the available resources (water, nutrients, and land) efficiently than other crop combinations (Du et al., 2018; Rahman et al., 2016; M. Raza et al., 2019). Therefore, it is the primary planting system for the production of maize and legume in the southwest of China (Liu et al., 2017; M. A. Raza et al., 2019). However, maize shading under this system significantly changes the light environment of the soybean canopy, especially the light intensity (photosynthetically active radiations) and light quality (R: FR ratio) of the soybean canopy (Liu et al., 2017; Yang et al., 2014). Light quality and light intensity are the two leading light characteristics in a shaded environment, which influences plant growth and development (Smith, 2000). Plants optimize photosynthesis to adapt to the decrease in light quantity and quality when the plants are exposed to shade (Gommers, Visser, St Onge, Voesenek, \& Pierik, 2013). In low light conditions, insufficient ATP for carbon fixation and carbohydrate biosynthesis leads to reduced plant growth (Shao et al., 2014). To avoid this situation, plants need to absorb light as much as possible to grow under low light conditions. However, under high-light conditions, plants need to make the most of their PSII capabilities to utilize sufficient light energy, and at the same time, deal with the excess photosynthetic capacity of sunlight (Mathur, Jain, \& Jajoo, 2018). Therefore, according to the nature of the light environment in which the plant is located, the plant can exhibit sun or shade leaves. Compared with shade leaves, sun leaves are small and thick, with well-developed palisade tissue, higher stomatal density, and thinner grana stacks (J. J. Casal, 2013). Shade leaves showed higher chlorophyll concentration per unit leaf mass and lower ATPase activity and Rubisco content compared to the sun (Martins et al., 2012). Photosynthesis organisms are subjected to changes in light quantity and light quality. They adjust their photosynthetic apparatus so that it maintains optimal performance under limiting light and minimizes photodamage under excess light (Rochaix, 2014). In vertical shade avoidance, increased stem and petiole growth improve leaf position (Kozuka et al., 2010). Exposure to low R: FR levels promote stem growth, but the perception of light signals by leaves contributes to this response (J. Casal \& Smith, 1988). When the plant is exposed to high irradiance, the chloroplast moves to the anticline wall of the palisade cell. This repositioning reduces the amount of light absorbed by the chloroplast and reduces damage to the photosynthetic apparatus (J. Casal \& Smith, 1988). Plant morphological and physiological characteristic exhibit plasticity in response to light intensity, and the leaf structure exhibit changes according to the light intensity (Hoshino, Yoshida, \& Tsukaya, 2019). Phenotypic plasticity tends to be low in shade-tolerant species; however, morphological features optimizing light capture can be high (Valladares \& Niinemets, 2008). To increase the light-capturing and distributing area, some plants modify their epidermal cells into a lens-like structure. Light capture changes with the angle, size, orientation, and photosynthetic apparatus (Mathur et al., 2018). In nature, many plants have a waxy coat on their leaf surface to avoid excessive sunlight (Osmond, 1993). Therefore, the plants have evolved to adopt mechanisms for optimum photosynthesis under changing light environments.

In previous studies, many researchers have investigated the impact of different light quantities and qualities on leaf size, structure, and movement (Feng et al., 2018; Yang et al., 2018); and they found that the leaf structure exhibits different features in the adaxial-abaxial according to the light intensity (Talbert \& Holch, 1957). Thick leaves formed under high light conditions are called 'sun leaves,' and thin leaves formed under low light conditions are called 'shade leaves' (Hoshino et al., 2019). Adjusting the thickness of the leaf can change the proportion of photosynthetic tissue per unit area of the leaf, thereby absorbing light energy more effectively (Terashima, Araya, Miyazawa, Sone, \& Yano, 2005). Furthermore, Sun and shade type of plants develops a respective type of chloroplasts which help plants to survive and perform photosynthesis under adverse conditions (Mathur et al., 2018). Photosynthesis is not only dependent on the quantity of light 
but also relies on the quality of light that reaches the chloroplasts, and photosynthetic responses to light are wavelength-dependent (Zhen \& van Iersel, 2017). The new study has shown that far-red light promotes photosynthesis in the low light stage of fluctuating light (Kono et al., 2020). Red light inhibits internode elongation, promotes lateral branching and tillering, and delays flower differentiation (Chen, Li, Zeng, Deng, \& Ren, 2019; Kreslavskii et al., 2013). Blue light mediates morphological and architectural acclimation of grape to shade and increases light capture (González et al., 2019). The morphological response of plants to shade changes the canopy structure, such as the three-dimensional distribution and orientation of stems, branches, and leaves.

Although the anatomical structure and physiological characteristics of the sun and shaded leaves have been studied in many plant species, it is still unclear how soybean leaves respond to shading from different shadetolerance perspectives. In this study, to clarify the effects on soybean plants grown under shading, we first performed shading treatment of soybean plants cultivated in the field and measured photosynthetic ability at approximately fifth trifoliolate (V5) stage, and the chlorophyll content and leaf structure after each shading treatment. To remove the influence of root interaction, we conducted a pot experiment on soybean plants with maize shading; and we recorded the same chlorophyll content, photosynthetic parameters, and leaf structure as we did in the field experiment.

\section{MATERIALS AND METHODS}

\subsection{Study site and plant materials}

Experiment 1

To evaluate the physiological response to shading of different shade-tolerance soybean plants, a field experiment, and a pot experiment were conducted. Two soybean cultivars with different shade-tolerance soybean plants to shade were employed in this research, high shade-tolerance: Nandou12 (ND12), low shade-tolerance: Guixia3 (GX3). The field experiments were conducted in 2018 at the Experimental Farm of Sichuan Agriculture University in Chongzhou City (30 $\left.56^{\prime} \mathrm{N}, 103 \operatorname{deg} 65^{\prime} \mathrm{E}\right)$, Sichuan province, China. The climate of the field was subtropical humid, with a mean annual temperature of 15.9, annual rainfall of $1012.4 \mathrm{~mm}$, annual average sunshine of $1161.5 \mathrm{~h}$, and a frost-free period of 285 days. The soybean cultivars ND12 and GX3, and the maize cultivar Chuandan 418 are used in this study. The intercropping patterns include maize-soybean relay strip intercropping (T1) and single row maize-soybean intercropping (T2) and sole soybean (CK) (Fig.1). Maize sowing and harvesting time were April 12, 2018, and August 15, 2018. Soybean sowing and harvesting time were June 17, 2018, and October 25, 2018.

$\mathrm{N}$ at the rate of $37.5 \mathrm{~kg} \mathrm{ha}^{-1}$ as urea, $\mathrm{P}$ at $600 \mathrm{~kg} \mathrm{ha}^{-1}$ as calcium, and $\mathrm{K}$ at $150 \mathrm{~kg} \mathrm{ha}^{-1}$ as potassium was applied to all treatments at the time of maize sowing. The second dose of $\mathrm{N}$ was applied at $75 \mathrm{~kg} \mathrm{ha}^{-1}, 150$ $\mathrm{kg} \mathrm{ha}^{-1}$ as urea, and $750 \mathrm{~kg} \mathrm{ha}^{-1}$ as ammonium bicarbonate at the seeding, jointing, and bell-mouthed stages of maize (Yang et al., 2017). $\mathrm{N}$ at $75 \mathrm{~kg} \mathrm{ha}^{-1}$ as urea, $\mathrm{P}$ at $600 \mathrm{~kg} \mathrm{ha}^{-1}$ as calcium superphosphate, and $\mathrm{K}$ at $60 \mathrm{~kg} \mathrm{ha}^{-1}$ as potassium chloride were applied at the time of soybean sowing, and the second dose of $\mathrm{N}$ was applied at $75 \mathrm{~kg} \mathrm{ha}^{-1}$ as urea at flowering stage of soybean (Yang et al., 2016).

Experiment 2

According to the results of light environment change in experiment 1, experiment 2 was conducted at in 2019 at the Teaching and Experiment Farm of Sichuan Agricultural University, Wenjiang, Sichuan province, China (30deg71'N, 103deg86'E). The climate of the experiment area was subtropical humid, with an annual temperature of 16, annual rainfall of $865.9 \mathrm{~mm}$, annual average sunshine of $991.1 \mathrm{~h}$, and a frost-free period of 282 days. Forty-eight rectangular plastic basins (43.5cm-length, $20 \mathrm{~cm}$-width and $14 \mathrm{~cm}$-height) were used in this experiment. Eight soybean seeds were sown in each basin. The basins were divided into three groups, sixteen basins in full sunlight (CK), sixteen basins in maize-soybean strip intercropping (T1), and sixteen basins in a single row maize-soybean intercropping (T2). The maize cultivars Chuandan418 (Zea mays L.) 
were selected for the experiment. Every soybean cultivar includes eight basins, different treatments described as follows in Fig.S1.

\subsection{Light environment}

Photosynthetically active radiation (PAR) and normalized photon flux were measured at the top of the soybean canopy (Fig.1, Fig.S1) using quantum sensors (Li-1500; America, LI-COR) and fiber optic spectrometer (Ava Spec-2048; Avantes, Netherlands) at the V5 stage of soybean. The sensors were placed horizontally 5 , $55,105,155$, and $205 \mathrm{~cm}$ above the soybean canopy (Liu et al., 2018). Normalized photon flux was measured at wavelengths ranging from $350 \mathrm{~nm}$ to $760 \mathrm{~nm}$. All treatments were measured between 11:00 and 13:00 on a sunny day (Kim, Lin, \& Mitchell, 2019).

(Figure 1.)

\subsection{Plant growth}

In this study, the plant height, biomass, and leaf traits of soybean were measured at the V5 stage under different light conditions. The biomass of the soybean plant was divided into stems, leaves, and petioles, dried in an oven at 105 for one hour, and dried to a constant weight at 75 to determine the total biomass of the leaves, petioles, and stems.

\subsection{Photosynthetic and fluorescence characteristics}

Photosynthetic parameters were determined of soybean fifth trifoliate leaves by a portable photosynthetic system (Li-6400, LI-COR, USA) on a sunny day from 9:00 am to 11:00 am. The net photosynthetic rate $\left(P_{n}\right.$ ), stomatal conductance $\left(G_{s}\right)$, and intercellular $\mathrm{CO}_{2}$ concentration $\left(C_{i}\right)$ were determined in a leaf chamber with an irradiance of $1000 \mu \mathrm{mol} \mathrm{m}{ }^{-2} \mathrm{~s}^{-1}$, the $\mathrm{CO}_{2}$ concentration of $400 \mu \mathrm{mol} \mathrm{mol}{ }^{-1}$, and the temperature was maintained at 25 (Kim et al., 2019).

Chlorophyll fluorescence of soybean leaves was determined by chlorophyll fluorescence image capture system (CFImager, Technologica, UK) and miniaturized pulse-amplitude-modulated system (MINI-PAM-2100, Walz, Germany). Each start measurement was performed at 9:00 and 22:00. Leaf about nine replications for each treatment. The effective photosystemII quantum yield ( $\varphi$ PSII), maximum photochemical efficiency $\left(F_{v} / F_{m}\right)$, PSII light capture efficiency $\left(F_{v}{ }^{\prime} / F_{m}{ }^{\prime}\right)$, PSII potential activity $\left(F_{v} / F_{0}\right)$, original fluorescence yield $\left(\mathrm{F}_{0}\right)$, non-photochemical quenching $(N P Q)$ and photochemical quenching $(q P)$ were analyzed.

\subsection{Leaf structure and chloroplast ultrastructure}

The soybean leaf piece $(5 \times 10 \mathrm{~mm})$ was fixed at 4 in formalin acetic acid alcohol (FAA, $70 \%$ alcohol $/ 38 \%$ formaldehyde/glacial acetic acid/, 90:5:5, v/v/v) solution embedded in paraffin. Light microscopy analysis (Nikon Eclipse 50i, Japan) was performed using a 10um thick transverse section of the leaf stained with fast green and counterstained with safranin. The thicknesses of the leaf tissues were obtained using ImageJ $1.42 \mathrm{q}$.

Leaves were cut into a $1 \mathrm{~mm} \times 2 \mathrm{~mm}$ piece and put into a $3 \%$ glutaraldehyde solution and fixed at 4 for one week. The sample was rinsed with a phosphoric acid buffer for four times, then transferred to $1 \%$ osmium tetroxide, fixed at 4 for 4 hours, rinsed with a phosphoric acid buffer for three times, dehydrated by acetone step by step, then transferred to Epon812 epoxy resin for soaking and embedding, polymerized at 60 for 24 hours. Ultrathin sections were cut with a diamond knife and stained with uranyl acetate and lead citrate. Sections were observed under a transmission electron microscope (TEM; HITACHI, H-600IV, Japan).

\subsection{Stomata characteristics}

Stomatal density was determined, followed the method of Xie Zhao sen et al. (Xie, Du, Li, \& Bondada, 2018). The leaves had become fully expanded; tissue clearing was applied to obtain the leaf segments. The 
replicas were observed under a light microscope (Nikon-E800), and a digital camera was used to photograph the replicas. The number of stomata was counted in six fields of view from the six marked leaves of six individual plants for each treatment.

\subsection{Statistical analysis}

All data analyses were presented by IBM SPSS Statistics (version 22.0). It was utilized to compare data through one or two-way ANOVA and test the differences among all treatments by a Tukey-Kramer comparison for significant differences at the 5\% level in all parameters. Microsoft Excel 2016 was used for data calculation. Origin 2018 and Adobe Illustrator 2020 were used to draw the figures.

\section{RESULTS}

\subsection{Changes in growth and development}

In this study, different shade and light treatments significantly changed the growth parameters of soybean cultivars (ND12 and GX3), and ND12 exhibited a stocky shape (Fig. 2b, c; Fig. S2b, c), while soybean cultivar GX3 showed a slender shape (Fig. 2e, f; Fig. S2e, f). Compared to CK treatment, the shade treatments T1 and T2 considerably increased the plant height of ND12 by $42 \%, 108 \%$, and GX3 by $44 \%$, $63 \%$. However, the maximum stem diameter $(8.4 \mathrm{~mm}$ and $6.43 \mathrm{~mm})$, aboveground biomass $(16.78 \mathrm{~g}$ and $11.9 \mathrm{~g})$, leaf area per plant $\left(455.15 \mathrm{~cm}^{2}\right.$ and $\left.268.38 \mathrm{~cm}^{2}\right)$, and specific leaf weight $(3.7 \mathrm{~g}$ and $4.3 \mathrm{~g})$ of ND12 and GX3, respectively, were noticed under CK. In contrast, all these parameters of both soybean cultivars were found minimum in $\mathrm{T} 1$ and $\mathrm{T} 2$ treatments, indicating that the growth parameters of soybean were directly associated with the availability of light.

(Figure 2.)

\subsection{Changes in gas exchange}

As shown in Fig.3 and Fig.S3, the highest values of photosynthetic rate $\left(P_{n} ; 20.19\right.$ and 17.53), stomatal conductance $\left(G_{s} ; 0.407\right.$ and 0.274$)$, and transpiration rate $\left(T_{r} ; 7.29\right.$ and 5.6$)$ of ND12 and GX3 soybean cultivars were recorded in CK treatment. In contrast, the lowest values of $P_{n}$ (7.05 and 4.79), $G_{s}(0.292$ and 0.069$)$, and $T_{r}$ (5.43 and 1.78) of ND12 and GX3 soybean cultivars were measured in T2 treatment. The intercellular $\mathrm{CO}_{2}$ concentrations $\left(C_{i}\right)$ of ND12 and GX3 in T1 and T2 treatments were increased as compared to CK treatment. Besides, in both soybean cultivars, the ND12 exhibited higher rates of $P_{n}(14.26)$, $G_{s}(0.306), T_{r}(5.90)$, and $C_{i}(248.37)$ than the corresponding values of $P_{n}(7.35), G_{s}(0.104), T_{r}(226.96)$, and $C_{i}(2.73)$ in GX3 in T1 treatment. These results show that shading could improve the photosynthetic gas exchange of soybean plants.

(Figure 3.)

\subsection{Changes in photosynthetic pigment content}

All treatments (T1, T2, and CK) significantly affected the Chl a, Chl b, and Car content of both soybean cultivars. The $\mathrm{Chl}$ a, $\mathrm{Chl} \mathrm{b}$, and Car content of soybean cultivars decreased with shading treatments $\mathrm{T} 1$ and T2 (Fig.4 and Fig.S4). Specifically, the Chl a, Chl b, Car content, and Chl a/b of GX3 under treatment T1 enhanced by $20.9 \%, 13.4 \%, 5.9 \%$, and $7.1 \%$, respectively, than the corresponding values of $\mathrm{Chl}$ a, Chl b, Car content and Chl a/b in ND12. However, there was no significant difference in the Chl a, Chl b, Car content, and $\mathrm{Chl} \mathrm{a/b}$ of GX3 and ND12 under treatment T2. In contrast, the highest ratio of Chl a/b in ND12 and GX3 (2.90 and 2.99 ) was calculated under CK treatment, while the lowest ratio of Chl a/b (2.56 and 2.45) was determined in treatments T2 (Fig.4d and Fig.S4d), respectively, indicating that the soybean plants under shade produce more $\mathrm{Chl} \mathrm{b}$ in a shading environment.

(Figure 4.) 


\subsection{Changes in chlorophyll fluorescence parameters}

In order to study the photosynthetic capacity of two different shade-tolerant soybean varieties ND12 and GX3. Measurements of chlorophyll fluorescence showed that the two soybean varieties increased the original fluorescence yield $\left(F_{o}\right)$ of photosystem II (PSII), reduce the maximum quantum yield PSII $\left(F_{v} / F_{m}\right)$, actual photosynthetic efficiency ( $\Phi$ PSII), and photosynthetic activity $\left(F_{v} / F_{0}\right)$ in a shaded environment. Under the T1 and T2 treatments, the $F_{o}$ of PSII in the ND12 was significantly lower than in the GX3 by $32 \%$ and $5.6 \%$ (Fig.5a and Fig.S5a). On the contrary, the $F_{v} / F_{m}$ of PSII in the ND12 $(0.852)$ were significantly higher than those in the GX3 (0.806) under the T1 treatment; no differences were noted for the CK treatments (Fig.5b and Fig.S5b). The non-photochemical quenching coefficient (NPQ) was the lowest of the shade treatments (Fig. 5e) in the field experiment but was no difference in the pot experiment (Fig. S5e). With the increase of shading (from T1 to T2 treatment), the $F_{v} / F_{0}$ of ND12 showed a trend of increasing first and then decreasing. However, the $F_{v} / F_{0}$ of GX3 always show a downward trend with increasing shade (Fig.5f and Fig.S5f).

(Figure 5.)

\subsection{Changes in leaf anatomy and chloroplast ultrastructure}

The results indicate that the leaf of soybean is a typical bifacial leaf, composed of palisade tissue, spongy tissue, and epidermal cells. Leaf slices revealed similarly sized and tightly arranged palisade tissue in the CK treatments. On the contrary, the T1 and T2 treated leaves had relatively loose palisade tissue cells and spongy tissue cells (Fig.6a'-f' and Fig.S6a'-f'). Differences in thickness of leaf, palisade tissue, sponge tissue, lower epidermal cell, and palisade cell area under the different shading treatments were observed in the following order: CK> T1 > T2 (Fig.6a,b,e,f and Fig.S6a,b,e,f). Under the T2 treatment, the thickness of the leaf and palisade cell area in the ND12 were significantly higher than in the GX3 by $15.9 \%$ and $3.4 \%$ (Fig.6a-c and Fig.S6a-c). On the contrary, in the field experiment, the thickness of leaf and palisade tissue of CK treatment in the ND12 were significantly lower than those in the GX3 by $11.8 \%$ and $17.2 \%$ (Fig.6a-b); no differences were noted for the CK treatments in the pot experiment (Fig.S6a-b). The thickness of the upper epidermal cell of the T2 treatment in the ND12 was significantly higher than in the GX3 in the field experiment (Fig.6d). There were no significant differences in the upper epidermal cell thickness among the treatments under the shading treatments in the pot experiment (Fig.S6d).

In order to observe the study of palisade tissue, peridermal sections passing through the first palisade layer were used (Fig.6g-i). The palisade cells of shading leaves (T1 and T2 treatments) revealed that in soybean exhibit cylindrical and they are loosely arranged, displaying big intercellular spaces compared with CK treatments. Palisade cells of ND12 and GX3 in T2 treatments are smaller than those of both CK treatments. Palisade cells of shading treatments in ND12 are more closely arranged than in GX3 (Fig.6h, I, $\mathrm{k}, \mathrm{l})$.

Chloroplasts contain chlorophyll for photosynthesis in plant cells. If chlorophyll synthesis is reduced, the chloroplast ultrastructure will change. The different shading treatments used in this research larger affected the chloroplast development in the leaves of soybean plants. The size and shape of the chloroplasts in the mesophyll cells were observed by transmission electron microscopy. In the CK treatment, the chloroplasts are fusiform shaped and are arranged along the plasma membrane. The structure of the grana is also clearly visible and extends parallel to the long axis, the thylakoids are closely arranged in an orderly manner, and as the shading increased, the grana gradually increased (Fig.7a', d' and Fig.S7a', d'). Chloroplasts were observed in the shading treatments (Fig.7c', f,' and Fig.S7c', f'). The chloroplast number per cell in the T2 treated grew the most. The chloroplasts of the T2 treated leaves were smaller, and the grana thickness of the thylakoids were increased. The grana thickness of the T1 treatment in the ND12 was significantly higher than in the GX3 by 14\% (Fig.7d). Otherwise, the starch grain area per chloroplast and starch-chloroplast area ratio of the T2 treatment in the ND12 was significantly lower than in the GX3 by $129 \%$ and $119 \%$ (Fig.7e-f). There were no significant differences in the chloroplast area and chloroplast circumference among the treatments under the shading treatments (Fig.7b, c, and Fig.S7b, c). 
(Figure 7.)

\subsection{Changes in stomatal morphology}

Each stomate is formed of two guard cells, which surround the stomatal pore. Plants can regulate gaseous exchange by dynamically controlling their pore apertures, which is the first response to environmental changes, including light intensity and water availability (Harrison, Arce Cubas, Gray, \& Hepworth, 2020). The stomatal size and stomatal density in GX3 varieties leave on soybean plants of the T2 treatment has declined significantly compared with the ND12 varieties (Fig.8 and Fig.S8); In the T1 and T2 treatments, leaves decreased the stomatal density by $8.7 \%$ and $27.3 \%$ on the abaxial surface in GX3 varieties leaves, compared with the ND12 varieties. These results suggest that stomatal density in GX3 varieties is mainly controlled by the light environment of leaves.

(Figure 8.)

\section{DISCUSSION}

\subsection{Shade response for growth and development of soybean}

The light environment is one of the most critical environmental factors that affect the growth and development of plants (Gao et al., 2020). Light gradients are omnipresent, so all plants are exposed to some degree of shade during their lifetime. The minimum light required for plant survival, shade tolerance, is an essential life-history feature that plays a crucial role in plant community dynamics (Valladares \& Niinemets, 2008). Shade tolerance refers to the ability of plants to tolerate low light. In this study, we found that the soybean seedlings exposure to shade treatments results in significantly higher plant height and stem biomass ratio and lower dry matter accumulation of aboveground compared with the soybean seedlings grown under the CK treatments. However, higher shade tolerance: ND12 variety, plant height is lower than GX3 (low shadetolerance varieties), but the leaf area, aboveground dry matter accumulation of ND12 is higher than GX3. The stem biomass allocation ratio of ND12 is lower than GX3, while the petiole biomass allocation ratio is higher than GX3. This suggests that the shade-tolerant soybean ND12 has lower phenotypic plasticity, while the shade-tolerant soybean GX3 has higher phenotypic plasticity than ND12. Shade tolerance is affected by plant individual development and many biological and abiotic factors. Although the phenotypic plasticity is lower in shade-tolerant species (e.g., lower elongation in low light), the plasticity of certain traits, especially the morphological features that optimize light capture, maybe high (Valladares \& Niinemets, 2008).

In this study, the intercropping shade resulted in a significant decrease in the leaf area of the two varieties of soybeans (Figure 2 and Figure S2), which reduced the individual's light energy absorption; moreover, the decline in leaf photosynthetic capacity was a direct limitation the synthesis of photosynthetic products (Figures 2 and 3). In this study, we also confirmed that the increase in the degree of shade could significantly reduce the biomass of a single soybean plant. However, the leaf area of different varieties of soybean decreased differently under the condition of intercropping (Figure 2). Obviously, the shade-intolerant soybean variety GX3 is more sensitive to the shade under the intercropping environment. The leaf area of GX3 was significantly higher than the ND12. Therefore, the reduction of leaf area per plant should be the main reason for soybean growth restriction.

\subsection{Shade response for photosynthetic and chlorophyll fluorescence of soybean}

The chloroplast is the place where plants photosynthesize, and chlorophyll content is often used to measure the light energy absorption of leaves (Pfundel, Latouche, Meister, \& Cerovic, 2018). Compared with plants grown under strong light, the chlorophyll content of leaves under artificial shading conditions is usually higher, which is conducive to plants in low light environments to enhance the absorption and capture of light energy by leaves, thereby maintaining photosynthesis (Li, Liu, Jiang, Liu, \& Shi, 2014). In this study, the canopy light intensity of the intercropping soybean population was low, which was reduced by about $60 \%$ (Figure 1), but the chlorophyll content of soybean leaves decreased (Figure 4). Previous studies on densely 
planted mint showed that under the environment of field root competition, dense planting and mutual shade could reduce the chlorophyll content of leaves. But when root competition in the field is eliminated, increasing the mutual shade of dense planting will cause the chlorophyll content of the leaves to increase (Tao \& Jiang, 2017). However, in this study, the chlorophyll content of soybean leaves decreased significantly under field intercropping conditions, while the decrease of chlorophyll content in the pot experiment was relatively small. It is my result that the similarity with this study appeared. Therefore, the competition of soybean roots for underground soil resources is intensified, and to a certain extent, the chlorophyll content of soybean leaves under low light is reduced, and the light energy absorption of the leaves is reduced. It also limits the ability of soybeans to use weak light. Chlorophyll is an important component of the PSII reaction center, so the chlorophyll content will affect the number of PSII reaction centers. The number of PSII reaction centers in leaves will decrease with the decrease of chlorophyll content. Therefore, the change in the number and function of Photosystem II may be an adaptation of soybean to the shade environment.

Photosystem II (PSII), as an important component of the photosynthetic machinery, is mainly related to the absorption, transmission, and transformation of light energy, and is very sensitive to environmental stress. A large number of studies have shown that under shade conditions, plants can increase the content of PSII antenna pigments to compensate for the reduced light energy capture due to the weakening of light intensity, thereby maintaining photosynthesis under low light (Gommers et al., 2013; Yamazaki \& Shinomiya, 2012; Yao et al., 2017). On the contrary, plants under strong light will reduce the content of PSII antenna pigments to avoid capturing too much light energy, and at the same time, enhance the excitation energy dissipation and antioxidant capacity to resist the damage of strong light to PSII and related photosynthetic institutions. Through the chlorophyll fluorescence parameters, we found that in the maize-soybean strip intercropping system, in addition to the increased the $F_{o}$ of the two soybean varieties, the $F_{v} / F_{m}$, $\Phi$ PSII, $q P$, NPQ, $F_{v} / F_{o}$ of the two soybean varieties were decreased, and the $F_{o}$ of GX3 under the shade of maize was significantly higher than that of ND12, while $F_{v} / F_{m}$ and $F_{v} / F_{o}$ were significantly lower than that of ND12. The $F_{o}$ of GX3 is significantly higher than that of ND12, which indicates that GX3 is more prone to light inhibition in the shaded environment. $F_{v} / F_{m}$ was the maximum light energy capture efficiency of PSII, indicating that the light energy capture per unit area of GX3 has decreased. However, the ratio of the captured light energy used for photochemical reactions ( PPSII) increased significantly in the field experiment (Figure 5). In the experiment, the heat dissipation (NPQ) of the leaves of the two soybean varieties did not change significantly. But in the pot experiment without root competition, soybean leaves dissipated more heat. Therefore, the competition of intercropping in the root system can stimulate the increase of heat dissipation.

\subsection{Shade response for gas exchange and leaf structure of soybean}

In the maize-soybean relay strip intercropping planting system, the high-lying crop maize causes severe shading of the soybean canopy, which significantly reduces the irradiance in the soybean canopy (Figure 1ef) and increases the far-red light (Figure 1h-i). Therefore, soybeans will produce shade leaves in this system (Figure 6b'-c', and e'-f'). We know that the morphological characteristics and anatomical structure of leaves play a key role in regulating photosynthetic capacity, providing a structural framework for gas diffusion and optimization of photosynthetic function (Gao et al., 2020; Li, Liu, Shi, \& Jiang, 2015). Theoretically, higher stomatal density and thicker leaves, as well as faster metabolite transfer between mesophyll cells, are conducive to a higher photosynthetic rate (Bertolino, Caine, \& Gray, 2019; Gotoh et al., 2018; Xiong, Huang, Peng, \& Li, 2017). Therefore, the stomatal density of GX3 leaves (Figure 8), the reduction of leaf thickness, and all the characteristics of the leaf morphology and anatomy (Figure 6) may be part of the reason for the reduced photosynthetic capacity in the intercropping system. It is well known that carbon dioxide enters the mesophyll tissue through the stomata on the surface of the leaf and is very sensitive to the light environment. In this study, maize shading under intercropping system planting conditions resulted in a significant decrease in soybean stomatal density and stomatal conductance (Figure 8). Therefore, the exchange of $\mathrm{CO}_{2}$ through the stomata may be restricted, resulting in a decrease in the absorption and transportation of $\mathrm{CO}_{2}$ in photosynthesis. 


\section{CONCLUSION}

In summary, under the maize-soybean relay strip intercropping planting system, the reduction of photosynthesis of soybeans involves the adjustment of leaf structure to light capture, the influence of stomatal characteristics on $\mathrm{CO}_{2}$ absorption and transportation, and the heat dissipation may play a role in soybeans grown in the field. Different shade-tolerant soybean varieties have significant differences in response to different degrees of shade. The shade-tolerant variety ND12 has advantages in the regulation of leaf structure and stomatal characteristics, which is more conducive to the progress of photosynthesis. Therefore, the shade-tolerant variety ND12 exhibited a higher photosynthetic capacity and PSII activity and biomass accumulation than variety GX3 in shade condition.

\section{ACKNOWLEDGEMENTS}

The research was funded by the National Nature Science Foundation (32071963), and Program on Industrial Technology System of National Soybean (CARS-04-PS19).

\section{CONF LICT OF INTEREST}

None declared.

\section{REFERENCES}

Bertolino, L. T., Caine, R. S., \& Gray, J. E. (2019). Impact of Stomatal Density and Morphology on Water-Use Efficiency in a Changing World. Front Plant Sci, 10 , 225. doi:10.3389/fpls.2019. 00225

Casal, J., \& Smith, H. (1988). The loci of perception for phytochrome control of internode growth in lightgrown mustard: Promotion by low phytochrome photoequilibria in the internode is enhanced by blue light perceived by the leaves. Planta, 176 , 277-282. doi:10.1007/BF00392456

Casal, J. J. (2013). Photoreceptor signaling networks in plant responses to shade. Annu Rev Plant Biol, 64 , 403-427. doi:10.1146/annurev-arplant-050312-120221

Chen, H., Li, Q. P., Zeng, Y. L., Deng, F., \& Ren, W. J. (2019). Effect of different shading materials on grain yield and quality of rice.Sci Rep, 9 (1), 9992. doi:10.1038/s41598-019-46437-9

Drinkwater, L., Wagoner, P., \& Sarrantonio, M. (1998). Drinkwater LE, Wagoner P, Sarrantonio M.. Legume-based cropping systems have reduced carbon and nitrogen losses. Nature 396: 262-265. Nature, 396 . doi: $10.1038 / 24376$

Du, J.-b., Han, T.-f., Gai, J.-y., Yong, T.-w., Sun, X., Wang, X.-c., . . . Yang, W.-y. (2018). Maize-soybean strip intercropping: Achieved a balance between high productivity and sustainability. Journal of Integrative Agriculture, 17 (4), 747-754. doi:10.1016/s2095-3119(17)61789-1

Feng, L., Raza, M. A., Li, Z., Chen, Y., Khalid, M. H. B., Du, J., . . . Yang, F. (2018). The Influence of Light Intensity and Leaf Movement on Photosynthesis Characteristics and Carbon Balance of Soybean.Front Plant Sci, 9 , 1952. doi:10.3389/fpls.2018.01952

Foley, J., Ramankutty, N., Brauman, K., Cassidy, E., Gerber, J., Johnston, M., . . . Zaks, D. (2011). Solutions for a Cultivated Planet.Nature, 478 , 337-342. doi:10.1038/nature10452

Gao, S., Liu, X., Liu, Y., Cao, B., Chen, Z., \& Xu, K. (2020). Photosynthetic characteristics and chloroplast ultrastructure of welsh onion (Allium fistulosum L.) grown under different LED wavelengths. BMC Plant Biol, 20 (1), 78. doi:10.1186/s12870-020-2282-0

Gommers, C. M., Visser, E. J., St Onge, K. R., Voesenek, L. A., \& Pierik, R. (2013). Shade tolerance: when growing tall is not an option. Trends Plant Sci, 18 (2), 65-71. doi:10.1016/j.tplants.2012.09.008 
González, C. V., Jeréz, D. N., Jofré, M. F., Guevara, A., Prieto, J., Mazza, C., . . . Giordano, C. V. (2019). Blue light attenuation mediates morphological and architectural acclimation of Vitis vinifera cv. Malbec to shade and increases light capture. Environmental and Experimental Botany, 157, 112-120. doi:10.1016/j.envexpbot.2018.09.023

Gotoh, E., Suetsugu, N., Higa, T., Matsushita, T., Tsukaya, H., \& Wada, M. (2018). Palisade cell shape affects the light-induced chloroplast movements and leaf photosynthesis. Sci Rep, 8 (1), 1472. doi:10.1038/s41598018-19896-9

Harrison, E. L., Arce Cubas, L., Gray, J. E., \& Hepworth, C. (2020). The influence of stomatal morphology and distribution on photosynthetic gas exchange. Plant J, 101 (4), 768-779. doi:10.1111/tpj.14560

Hoshino, R., Yoshida, Y., \& Tsukaya, H. (2019). Multiple steps of leaf thickening during sun-leaf formation in Arabidopsis. Plant J, 100 (4), 738-753. doi:10.1111/tpj.14467

Kim, H.-J., Lin, M.-Y., \& Mitchell, C. A. (2019). Light spectral and thermal properties govern biomass allocation in tomato through morphological and physiological changes. Environmental and Experimental Botany, 157 , 228-240. doi:10.1016/j.envexpbot.2018.10.019

Kono, M., Kawaguchi, H., Mizusawa, N., Yamori, W., Suzuki, Y., \& Terashima, I. (2020). Far-Red Light Accelerates Photosynthesis in the Low-Light Phases of Fluctuating Light. Plant Cell Physiol, 61 (1), 192-202. doi:10.1093/pcp/pcz191

Kozuka, T., Kobayashi, J., Horiguchi, G., Demura, T., Sakakibara, H., Tsukaya, H., \& Nagatani, A. (2010). Involvement of Auxin and Brassinosteroid in the Regulation of Petiole Elongation under the Shade.Plant Physiology, 153 , 1608-1618. doi:10.1104/pp.110.156802

Kreslavskii, V., Lyubimov, V., Shirshikova, G., Shmarev, A., Kosobryukhov, A., Schmitt, F.-J., . . . Allakhverdiev, S. (2013). Preillumination of lettuce seedlings with red light enhances the resistance of photosynthetic apparatus to UV-A. Journal of photochemistry and photobiology. B, Biology, 122C , 1-6. doi:10.1016/j.jphotobiol.2013.02.016

Li, T., Liu, L. N., Jiang, C. D., Liu, Y. J., \& Shi, L. (2014). Effects of mutual shading on the regulation of photosynthesis in field-grown sorghum. $J$ Photochem Photobiol B, 137, 31-38. doi:10.1016/j.jphotobiol.2014.04.022

Li, T., Liu, Y., Shi, L., \& Jiang, C. (2015). Systemic regulation of photosynthetic function in field-grown sorghum. Plant Physiol Biochem, 94 , 86-94. doi:10.1016/j.plaphy.2015.05.008

Liu, X., Rahman, T., Song, C., Su, B., Yang, F., Yong, T., . . Yang, W. (2017). Changes in light environment, morphology, growth and yield of soybean in maize-soybean intercropping systems. Field Crops Research, 200 , 38-46. doi:10.1016/j.fcr.2016.10.003

Liu, X., Rahman, T., Song, C., Yang, F., Su, B., Cui, L., . . Y Yang, W. (2018). Relationships among light distribution, radiation use efficiency and land equivalent ratio in maize-soybean strip intercropping.Field Crops Research, 224, 91-101. doi:10.1016/j.fcr.2018.05.010

Martins, S., Detmann, K., Reis, J., Pereira, L., Sanglard, L., Rogalski, M., \& DaMatta, F. (2012). Photosynthetic induction and activity of enzymes related to carbon metabolism: insights into the varying net photosynthesis rates of coffee sun and shade leaves. Theoretical and Experimental Plant Physiology, 25 , 62-69. doi:10.1590/S2197-00252013000100008

Mathur, S., Jain, L., \& Jajoo, A. (2018). Photosynthetic efficiency in sun and shade plants. Photosynthetica, 56 (1), 354-365. doi:10.1007/s11099-018-0767-y

Osmond, S. A. R. C. E. L. a. C. B. (1993). Wax as a Mechanism for Protection against photoinhibition-A study pf Cotyledon orbiculata.pdf. Plant Biology, 106,5 . 
Pfundel, E. E., Latouche, G., Meister, A., \& Cerovic, Z. G. (2018). Linking chloroplast relocation to different responses of photosynthesis to blue and red radiation in low and high light-acclimated leaves of Arabidopsis thaliana (L.). Photosynth Res, 137 (1), 105-128. doi:10.1007/s11120-018-0482-3

Rahman, T., Ye, L. I. N., Liu, X. I. N., Iqbal, N., Du, J., Gao, R., . . Yang, W. (2016). WATER USE EFFICIENCY AND WATER DISTRIBUTION RESPONSE TO DIFFERENT PLANTING PATTERNS IN MAIZE-SOYBEAN RELAY STRIP INTERCROPPING SYSTEMS. Experimental Agriculture, -1 , 1-19. doi:10.1017/S0014479716000260

Raza, M., Khalid, M. H., Zhang, X., Feng, L., Khan, I., Hassan, M., . . Y Yang, W. (2019). Effect of planting patterns on yield, nutrient accumulation and distribution in maize and soybean under relay intercropping systems. Scientific Reports, 9 . doi:10.1038/s41598-019-41364-1

Raza, M. A., Bin Khalid, M. H., Zhang, X., Feng, L. Y., Khan, I., Hassan, M. J., . . . Yang, W. (2019). Effect of planting patterns on yield, nutrient accumulation and distribution in maize and soybean under relay intercropping systems. Sci Rep, 9 (1), 4947. doi:10.1038/s41598-019-41364-1

Rochaix, J. D. (2014). Regulation and dynamics of the light-harvesting system. Annu Rev Plant Biol, 65 , 287-309. doi:10.1146/annurev-arplant-050213-040226

Shao, Q., Wang, H., Guo, H., Zhou, A., Huang, Y., Sun, Y., \& Li, M. (2014). Effects of Shade Treatments on Photosynthetic Characteristics, Chloroplast Ultrastructure, and Physiology of Anoectochilus roxburghii.PLoS One, 9 , e85996. doi:10.1371/journal.pone.0085996

Smith, H. (2000). Phytochromes and light signal perception by plants - An emerging synthesis. Nature, 407 , 585-591. doi:10.1038/35036500

Talbert, C., \& Holch, A. (1957). A Study of the Lobing of Sun and Shade Leaves. Ecology, 38 , 655. doi:10.2307/1943135

Tao, L. I., \& Jiang, C. D. (2017). Effects of close planting on photosystem II functions in Mentha haplocalyx leaves. Plant Physiology Journal .

Terashima, I., Araya, T., Miyazawa, S.-I., Sone, K., \& Yano, S. (2005). Construction and Maintenance of the Optimal Photosynthetic Systems of the Leaf, Herbaceous Plant and Tree: an Eco-developmental Treatise.Annals of botany, 95 , 507-519. doi:10.1093/aob/mci049

Valladares, F., \& Niinemets, U. (2008). Shade Tolerance, a Key Plant Feature of Complex Nature and Consequences. Annual Review of Ecology, Evolution, and Systematics, 39 (1), 237-257. doi:10.1146/annurev.ecolsys.39.110707.173506

Xie, Z.-S., Du, H.-R., Li, J.-B., \& Bondada, B. (2018). Morphological and structural changes of stomata and leaf veins during growth of grape leaves using tissue clearing technique. Zhiwu Shengli Xuebao/Plant Physiology Journal, 54 (2), 237-246.

Xiong, D., Huang, J., Peng, S., \& Li, Y. (2017). A few enlarged chloroplasts are less efficient in photosynthesis than a large population of small chloroplasts in Arabidopsis thaliana. Sci Rep, 7 (1), 5782. doi:10.1038/s41598-017-06460-0

Yamazaki, J., \& Shinomiya, Y. (2012). Effect of partial shading on the photosynthetic apparatus and photosystem stoichiometry in sunflower leaves. Photosynthetica, 51 (1), 3-12. doi:10.1007/s11099-012-0073z

Yang, F., Feng, L., Liu, Q., Wu, X., Fan, Y., Raza, M. A., . . . Yang, W. (2018). Effect of interactions between light intensity and red-to- far-red ratio on the photosynthesis of soybean leaves under shade condition. Environmental and Experimental Botany, 150 , 79-87. doi:10.1016/j.envexpbot.2018.03.008

Yang, F., Huang, S., Gao, R., Liu, W., Yong, T., Wang, X., . . . Yang, W. (2014). Growth of soybean seedlings in relay strip intercropping systems in relation to light quantity and red:far-red ratio. Field Crops 
Research, 155 , 245-253. doi:10.1016/j.fcr.2013.08.011

Yang, F., Liao, D., Wu, X., Gao, R., Fan, Y., Raza, M. A., . . . Yang, W. (2017). Effect of aboveground and belowground interactions on the intercrop yields in maize-soybean relay intercropping systems. Field Crops Research, 203 , 16-23. doi:10.1016/j.fcr.2016.12.007

Yang, F., Ying, L., Liu, Q. L., Fan, Y. F., Liu, W. G., Yong, T. W., . . . Yang, W. Y. (2016). Effect of Maize Row Spacing on Biomass, Root Bleeding Sap and Nutrient of Soybean in Relay Strip Intercropping Systems. Scientia Agricultura Sinica .

Yao, X., Li, C., Li, S., Zhu, Q., Zhang, H., Wang, H., . . . Xie, F. (2017). Effect of shade on leaf photosynthetic capacity, light-intercepting, electron transfer and energy distribution of soybeans. Plant Growth Regulation, 83 (3), 409-416. doi:10.1007/s10725-017-0307-y

Zhen, S., \& van Iersel, M. W. (2017). Far-red light is needed for efficient photochemistry and photosynthesis. J Plant Physiol, 209 , 115-122. doi:10.1016/j.jplph.2016.12.004 